\title{
Retraction Note: Downregulation of microRNA-324-3p inhibits lung cancer by blocking the NCAM1-MAPK axis through ALX4
}

Published online: 10 June 2021

(c) The Author(s), under exclusive licence to Springer Nature America, Inc. 2021

Retraction to: Cancer Gene Therapy

https://doi.org/10.1038/s41417-020-00231-2

Published online 21 October 2020

The Editor-in-Chief has retracted this Article on request from the corresponding author who reported that the results presented in the article are not reproducible. The
Editor-in-Chief no longer has confidence in the reliability of the data underlying this article.

Tieniu Song agrees to this retraction, but has not responded to correspondence about this retraction wording. Hui Zhou, Xiaoping Wei, Yuqi Meng, and Quanwei Guo have not responded to any correspondence from the Publisher about this retraction. 